Research Article

\title{
Type 2 Diabetes Mellitus and Chronic Heart Failure with Midrange and Preserved Ejection Fraction: A Focus on Serum Biomarkers of Fibrosis
}

\author{
D. A. Lebedev $(\mathbb{D}$, E. A. Lyasnikova $\mathbb{D}$, E. Yu Vasilyeva $\mathbb{D}$, A. Yu Babenko $\mathbb{D}$, \\ and E. V. Shlyakhto \\ Almazov National Medical Research Centre, Saint-Petersburg, Russia \\ Correspondence should be addressed to D. A. Lebedev; doctorlebedev11@gmail.com
}

Received 22 July 2020; Revised 12 October 2020; Accepted 28 October 2020; Published 9 November 2020

Academic Editor: Gaetano Santulli

Copyright $\odot 2020$ D. A. Lebedev et al. This is an open access article distributed under the Creative Commons Attribution License, which permits unrestricted use, distribution, and reproduction in any medium, provided the original work is properly cited.

\begin{abstract}
As myocardial fibrosis might be an important contributor to the association of diabetes mellitus with left ventricular (LV) dysfunction and chronic heart failure (HF), we investigated the profile of some proinflammatory, profibrotic biomarkers in patients with type 2 diabetes mellitus (T2DM) at various stages of the cardiovascular disease continuum from absence of clinic since and symptoms to HF with preserved (HFpEF) and midrange ejection fraction (HFmrEF). Material and Methods. Sixty-two patients with T2DM (age 60 [55; 61]), 20 patients without clinical manifestations of HF and 2 groups with clinical manifestations of stable HF, 29 patients with HFpEF, and 13 patients with HFmrEF, were included in the study. The control group consisted of 13 healthy subjects and normal BMI. All patients underwent transthoracic echocardiography, laboratory assessment of $\mathrm{N}$-terminal fragment of the brain natriuretic peptide (Nt-proBNP), highly sensitive C-reactive protein (hsCRP), soluble suppression of tumorigenesis-2 (sST2), galectin-3, C-terminal propeptide of procollagen type I (PICP), N-terminal propeptide of procollagen type III (PIIINP), matrix metalloproteinase-9 (MMP-9), and tissue inhibitor of matrix proteinase-1 (TIMP-1). Results. Patients with HFmrEF had higher values of LV volumetric parameters, indexed parameters of LV myocardial mass (LVMM), and higher concentrations of Nt-proBNP (all $p<0.05)$. The concentrations of galectin-3 were greater in patients with HFpEF and HFmrEF compared to patients without HF ( $p=0.01$ and $p=0.03$, respectively). PICP and PICP/PIIINP ratio were greater in patients with HFmrEF compared to patients with $\operatorname{HFpEF}(p=0.043$ and $p=0.033$, respectively). In patients with T2DM and HF, a relationship was found between galectin-3 and LVMM/body surface area $(r=-0.58, p=0.001)$, PIIINP, TIMP-1, and LV end-diastolic volume $(r=-0.68$ and $p=0.042$ and $r=0.38$ and $p=0.02$, respectively). Conclusion. The dynamics at various stages of the cardiovascular disease continuum in the serum fibrosis markers may reflect an increase in fibrotic and decrease in antifibrotic processes already at the preclinical stage of HF. At the same time, the changes found in the circulating procollagen levels may indicate a shift in balance towards type I collagen synthesis in HFmrEF compared with HFpEF.
\end{abstract}

\section{Introduction}

Chronic heart failure (HF) and type 2 diabetes mellitus (T2DM) are important problems of most national health systems. Patients with T2DM have a high risk of developing $\mathrm{HF}$, and in a quarter of HF patients with reduced (HFrEF), midrange (HFmrEF), and preserved ejection fraction (EF) (HFpEF) already had T2DM, which significantly worse the prognosis of the disease, increasing mortality by $30-50 \%$. $[1,2]$.
Taking into account the clinical phenotypic heterogeneity of HFpEF, its pathogenetic mechanisms have not been fully determined. At the same time, the basic characteristics, pathophysiology, and approaches to the treatment of patients with HFmrEF are being actively studied. The pathophysiology of cardiovascular diseases, including HF, in T2DM is multifactorial. T2DM is one of the most significant noncardiac diseases in which a proinflammatory and profibrotic state leads to the development of interstitial myocardial 
fibrosis, increase in stiffness of myocardium, and a deterioration in elasticity. Also, insulin resistance promotes development of vascular stiffness and hypertension, dyslipidemia, and increased systemic inflammation. Elevated glucose levels lead to increased formation of advanced glycation endproducts and accumulation of reactive oxygen species [3]. In combination with visceral obesity, T2DM with a high frequency causes diastolic dysfunction and HF [4]. Hyperglycemia inevitably deteriorates profibrotic processes associated with oxidative, hemodynamic, and metabolic stress in HF [3]. Diabetic cardiomyopathy is usually associated with arterial hypertension $(\mathrm{AH})$ and ischemic heart disease (IHD), forming the so-called "mixed" clinical phenotype of HF, which may be present with both HFpEF and HFmrEF [5]. For specification of pathogenesis of different phenotypes of $\mathrm{HF}$, research of different circulating biomarkers and their diagnostic and prognostic values seem perspective.

Much attention is paid to markers of myocardial stress, such as the N-terminal fragment of brain natriuretic peptide (Nt-proBNP) and markers of inflammation; the most available and studied from which is the highly sensitive Creactive protein (hsCRP). However, markers associated with profibrotic processes are of particular interest too. Such markers include sST2 (stimulating factor growth expression gene 2, soluble form, also known as IL1RL1, and suppression of tumorigenicity 2), galectin-3, type I procollagen Cterminal propeptide (PICP) and type III procollagen $\mathrm{N}$ terminal propeptide (PIIINP), matrix metalloproteinases (MMP), and their tissue inhibitors (TIMP) [6]. The role of each of these biomarkers is rather specific, and identification of their role in patients with T2DM is complicated by a row of factors. Firstly, T2DM usually occurs in patients with visceral obesity, which is characterized by chronic inflammation and oxidative stress. Thus, it is rather difficult to separate the effects of obesity on biomarker levels from the effects of T2DM itself. Secondly, most of the biomarkers circulating in the blood in HF can be influenced by various factors, including body mass index (BMI) and glycemic levels. Comparison of the serum biomarker levels in patients with similar $\mathrm{BMI}$ and similar glycemic control without $\mathrm{HF}$ and with HFpEF and HFmrEF will clarify these issues. It should be noted that the relationship between diabetes-related fibrosis and phenotype of HF has not been systematically studied. In addition, little has been studied about difference in patients with T2DM and HFpEF or HFmrEF. In this study, we evaluate fibrosis biomarkers in patients with T2DM and HFpEF or HFmrEF and compare their clinical characteristics and structural and functional parameters of myocardial remodeling.

\section{Materials and Methods}

All procedures were carried out in accordance with the Declaration of Helsinki. The Ethical Committee of the Almazov National Medical Research Centre approved the study (№020419). All patients provided written informed consent. Sixty-two patients with T2DM were included in the study: 20 patients without clinical signs and symptoms of HF (group 1) and 2 groups with clinical manifestations of stable HF: 29 patients with HFpEF (group 2) and 13 patients with $\mathrm{HFmrEF}$ (group 3). There was also a control group, which consisted of 13 healthy subjects comparable in age and sex with the studied cohorts, with normal BSA $\left(22-25 \mathrm{~kg} / \mathrm{m}^{2}\right)$ and heart function. The inclusion criteria in the study were age 40 to 75 years, T2DM, glycated hemoglobin (HbA1c) 7.0-11.0\%, and stable hypoglycemic therapy for at least 12 weeks prior to study. In addition, for patients with $\mathrm{HF}$, inclusion criteria were the presence of stable HF (II functional class (NYHA)), HFpEF or HFmrEF, and optimal drug therapy for $\mathrm{HF}$ for at least 3 months. The exclusion criteria were valvular heart disease, cardiomyopathy, rheumatological diseases, significant cardiovascular events, including acute myocardial infarction (MI) or major heart surgery, percutaneous coronary intervention (PCI), valvuloplasty within 12 months, and hospitalization due to decompensation of HF within 3 months prior to enrollment in the study; planned intervention on the coronary arteries, HFrEF, III-IV functional class (NYHA), and glomerular filtration rate (GFR) according to Chronic Kidney Disease Epidemiology Collaboration Formula $(\mathrm{CKD}-\mathrm{EPI})<60 \mathrm{ml}$ $/ \mathrm{min} / 1.73 \mathrm{~m}^{2}$; obesity of the III degree; severe bronchial asthma or chronic obstructive pulmonary disease; and secondary arterial hypertension, blood pressure $(\mathrm{BP})>180$ $/ 110 \mathrm{mmHg}$, and severe liver disease. The diagnosis of HFpEF and HFmrEF was made according to the recommendations of the European Society of Cardiology 2016 [7]. Laboratory tests were performed, including $\mathrm{HbA1c}$, lipids, and blood creatinine with calculation of GFR, ECG, and echocardiography (VIVID 9 GE, USA) according to a standard protocol by one blinded operator. The left ventricular myocardium mass (LVM) was additionally indexed to the height (in meters). Using height-based indexing ( $\mathrm{LVM} /$ height in $\mathrm{m}^{2.7}$ ) in individuals with $\mathrm{BMI} \geq 30 \mathrm{~kg} / \mathrm{m}^{2}$ is preferred in order to avoid underestimation of the prevalence of LV hypertrophy in obese patient $[8,9]$. LV hypertrophy was defined using genderspecific criteria with LVM corrected for BSA, height, and height raised to the allometric power of 2.7 according to ASE/EACVI-2015 recommendations on the use of echocardiography in adult hypertension [10]. Intraoperator variability for the assessment of volumetric echocardiographic parameters in our research centre was less than $4 \%$. Longitudinal systolic myocardial deformation was assessed by $2 \mathrm{D}$ speckle tracking echocardiography. The automated functional imaging (AFI) software was used for the analysis. The maximum longitudinal strain (GLS) of left ventricle (LV) was calculated by averaging the peak systolic values of 18 segments obtained from 6 segments of three apical views (four-chamber, twochamber, and long axis of LV). The panel of studied biomarkers included Nt-proBNP, hsCRP, sST2, galectin-3, PICP, PIIINP, MMP-9, and TIMP-1. The serum samples were frozen at $-80^{\circ} \mathrm{C}$ until analysis. The serum hsCRP level was determined on an automatic biochemical analyzer "Cobas Integra $400+$ " by the immunoturbidimetric method. Serum NtproBNP concentration was assessed by electrochemiluminescence method using the Elecsys test system (Roche Diagnostic). Serum levels of galectin-3 (R\&D system), MMP-9 and TIMP1 (R\&D system), sST2 (Clinical diagnostics, Presage ST2 kit), and PICP and PIIINP (USCN Life Science) were assessed by enzyme immunoassay. Additionally, the ratios of 
MMP-9/TIMP1 and PICP/PIIINP were calculated for each patient. Echocardiography and blood sampling for biomarkers were performed on the background of sinus rhythm in all patients.

2.1. Statistical Analysis. Statistical analysis was performed using the SPSS statistical software (version 21.0, IBM Corp, USA). Continuous variables were presented as medians with 25 th to 75 th interquartile ranges, and categorical variables were presented as percentages. Continuous variables were compared by Kruskal-Wallis tests followed by Mann-Whitney $U$ test when appropriate. Correlations were analysed using Spearman's rank correlation tests. Categorical variables were compared by chi-squared test. Statistical significance was defined as $p$ value $<0.05$.

\section{Results}

Table 1 shows the main characteristics of the groups. The studied groups had comparable clinical and demographic characteristics. The median age in all groups was about 60 years; the proportion of men was more than $50 \%$. AH was observed in all patients of the studied groups. The duration of $\mathrm{AH}$ and the level of office BP did not differ between the groups. Overweight or obesity was diagnosed in all patients. There were no significant differences in BMI and T2DM duration, low-density lipoproteins (LDL), and triglycerides (TG) levels between the groups. HbAlc concentrations in the group without HF, in the group with HFpEF, and in the group with HFmrEF were $8.3 \%, 8.5 \%$, and $8.8 \%$, respectively $(p>0.05)$. There were no significant gender differences, duration of HF and T2DM, the presence of concomitant diseases, and drug therapy in groups 2 and 3 . More than $40 \%$ of patients between groups 2 and 3 underwent myocardial revascularization. The incidence of paroxysmal atrial fibrillation among patients with HF varied slightly (15-17\%). An endocrinologist and a heart failure specialist consulted all patients with HF. Patients in groups 2 and 3 received the recommended therapy for HF: 77-90\% angiotensin-converting enzyme inhibitors (ACEi) or angiotensin receptor antagonists (ARA), 77-90\% beta-blockers, 55-61\% mineralocorticoid receptor antagonists (MRA), and 100\% diuretics. Combined antihyperglycemic therapy in all groups was represented by metformin, sulfonylureas, and/or dipeptidyl peptidase-4 inhibitors (DPP-4).

3.1. Echocardiographic Parameters. Echocardiography data are presented in Table 2. Patients with HFmrEF had significantly lower LV EF and lower values of GLS LV compared to patients with HFpEF and patients without HF $(p 1.2<0.05)$. There was an increase in the left atrial volume index (LA volume index) in patients with HF. This increase was combined with grade 1 diastolic dysfunction in $30 \%$ of HF patients. In most cases, patients of all groups had concentric LV myocardial hypertrophy, characterized by an increase in the indexed values of LVM and relative wall thickness (RWT) of the LV. Patients with HFmrEF had significantly higher values of LV end-diastolic volume (EDV) and LV volume index compared to patients with HFpEF and patients without HF (all $p<0.05$ ). The indexed parameters of LV $\mathrm{MM}$ increased in patients with $\mathrm{HF}$, and their highest values were observed in patients with HFmrEF.

3.2. Molecular Biomarkers. Data on the concentrations of biomarkers are presented in Table 3. Lower (50\% lower) threshold serum concentrations of Nt-proBNP were used in patients with clinical manifestations of HF, echocardiographic criteria, and BMI $>30 \mathrm{~kg} / \mathrm{m}^{2}$, taking into account the high incidence of obesity in the study sample [8]. Higher Nt-proBNP concentrations were observed in the group of HFmrEF $(p<0.05)$. The sST- 2 concentrations did not significantly differ between the investigated groups and the control group. The concentrations of galectin-3, PICP, PIIINP, and the PICP/PIIINP ratio were significantly higher in all investigated groups compared with the control group (all $p<0.05$ ). The concentrations of galectin-3 were greater in patients with HFpEF and HFmrEF compared to patient without HF ( $p=0.01$ and $p=0.03$, respectively). There was a negative correlation between galectin-3 and high-density lipoprotein (HDL) $(r=-0.542 ; p=0.004)$, which confirmed the participation of this biomarker in the process of atherogenesis. Higher PICP concentrations and the PICP/PIIINP ratio were observed in the group of HFmrEF and in the group without HF compared to the control group. PICP and PICP/PIIINP ratio were greater in patients with $\mathrm{HFmrEF}$ compared to patients with $\operatorname{HFpEF}$ ( $p=0.043$ and $p=0.033$, respectively). There was a decrease in the serum concentration of MMP-9 in the groups with HF compared to the control group, although did not quite achieve the threshold for statistical significance. At the same time, the TIMP-1 levels between the studied groups did not significantly differ. The MMP9/TIMP-1 ratio was significantly lower in all investigated groups compared to the control group (all $p<0.05$ ). Furthermore, the decrease in the MMP-9/TIMP-1 ratio was more pronounced in patients with HF. There were no differences in the concentrations of hsCRP, galectin-3, PIIINP, MMP9, TIMP-1, and the MMP-9/TIMP-1 ratio between patients with HFpEF and HFmrEF. There were no correlations between circulating biomarkers and gender, age, $\mathrm{HbAlc}$, $\mathrm{BMI}$, and drug therapy. There were positive associations between Nt-proBNP concentrations and LA volume index $(r=0.44 ; p=0.003)$, galectin-3, and values of left ventricular myocardial mass/body surface area (LVM/BSA) $(r=-0.58$, $p=0.001)$, PIIINP and LV EDV $(r=0.38 ; p=0.02)$, and negative association between TIMP-1 concentrations and LV EDV $(r=-0.68 ; p=0.042)$ in groups with HF.

\section{Discussion}

The list of potential mechanisms contributing to the development of HF in T2DM patients continues to expand each decade. The main processes include oxidative stress, inflammation, impaired sensitivity to insulin, metabolic abnormalities, such as calcium exchange in the myocardium, dysfunction of mitochondria and endothelium, neurohumoral activation, and death of cardiomyocytes. It is not the full list of the difficult processes mediated by hyperglycemia, leading to hypertrophy, excess diffuse extracellular matrix formation, 
TABLE 1: Demographic and clinical characteristics of the patients.

\begin{tabular}{|c|c|c|c|c|}
\hline Parameters & $\begin{array}{l}\text { Group } 1 \text { without } \operatorname{HF}(n=20) \\
\operatorname{Me}(25,75)\end{array}$ & $\begin{array}{c}\text { Group } 2 \operatorname{HFpEF}(n=29) \\
\operatorname{Me}(25,75)\end{array}$ & $\begin{array}{c}\text { Group } 3 \text { HFmrEF }(n=13) \\
\operatorname{Me}(25,75)\end{array}$ & $\begin{array}{l}p \text { for groups } 1,2, \\
\text { and } 3\end{array}$ \\
\hline Age, years & $57(52,60)$ & $61(57,66)$ & $59(54,62)$ & 0.44 \\
\hline Gender, male, $n(\%)$ & $13(65)$ & $15(51.7)$ & $7(53.9)$ & 0.74 \\
\hline Duration of CHF, years & - & $9(6,13)$ & $8(5,11)$ & 0.68 \\
\hline $\mathrm{CAD}, n(\%)$ & - & $20(68.9)$ & $11(84.6)$ & 0.71 \\
\hline MI, $n(\%)$ & - & $15(51.7)$ & $7(63.6)$ & 0.85 \\
\hline CABG/PCI, $n(\%)$ & - & $14(48.2)$ & $7(63.6)$ & 0.22 \\
\hline Stable angina II, $n(\%)$ & - & $11(37.9)$ & $6(46.1)$ & 0.62 \\
\hline $\begin{array}{l}\text { Duration of hypertension, } \\
\text { years }\end{array}$ & $14(9,18)$ & $18(13,22)$ & $16(11 ; 19)$ & 0.19 \\
\hline $\mathrm{SBP}, \mathrm{mmHg}$ & $130(115,140)$ & $140(110,155)$ & $130(110,145)$ & 0.76 \\
\hline $\mathrm{DBP}, \mathrm{mmHg}$ & $85(75,90)$ & $80(65,90)$ & $75(60,85)$ & 0.62 \\
\hline Heart rate, $\mathrm{b} / \mathrm{min}$ & $77(68,90)$ & $70(62,78)$ & $68(63,74)$ & 0.29 \\
\hline $\begin{array}{l}\text { Atrial fibrillation, paroxysmal } \\
\text { form, } n(\%)\end{array}$ & - & $5(17.2)$ & $2(15,3)$ & 0.31 \\
\hline Duration of diabetes, years & $10(6,13)$ & $12(9,16)$ & $13(5,17)$ & 0.14 \\
\hline BMI, $\mathrm{kg} / \mathrm{m}^{2}$ & $32.8(30.2,34.9)$ & $32.3(28.7,36.8)$ & $34.7(31.7,37.8)$ & 0.38 \\
\hline Overweight, $n(\%)$ & $3(15)$ & $7(24.1)$ & $2(15.4)$ & 0.67 \\
\hline Obesity I degree, $n(\%)$ & $12(60)$ & $17(58.6)$ & $7(53.8)$ & 0.9 \\
\hline Obesity II degree, $n(\%)$ & $5(25)$ & $5(17.3)$ & $4(30.8)$ & 0.59 \\
\hline Smoking, $n(\%)$ & $10(50)$ & $13(44.8)$ & $6(46.1)$ & 0.76 \\
\hline Hemoglobin, g/l & $144(128,157)$ & $140(125,159)$ & $138(120,151)$ & 0.53 \\
\hline $\mathrm{HbA}_{1 \mathrm{c}}, \%$ & $8.3(7.7,9.3)$ & $8.5(7.9,9.7)$ & $8.8(8.4,9.9)$ & 0.49 \\
\hline $\mathrm{GFR}, \mathrm{ml} / \mathrm{min} / 1.73 \mathrm{~m} 2$, & $98(82,109)$ & $93(74,105)$ & $86(68,96)$ & 0.52 \\
\hline $\mathrm{LDL}, \mathrm{mmol} / \mathrm{l}$ & $2.7(2.0,3.7)$ & $2.1(1.3,3.3)$ & $1.9(1.1,2.8)$ & 0.65 \\
\hline $\mathrm{HDL}, \mathrm{mmol} / \mathrm{l}$ & $1.15(0.87,1.28)$ & $1.12(0.91,1.35)$ & $0.96(0.82,1.33)$ & 0.63 \\
\hline Triglycerides, mmol/l & $2.4(1.8,3.2)$ & $2.2(1.7,2.9)$ & $2.6(2.1,3.3)$ & 0.19 \\
\hline ACEi/ARA, $n(\%)$ & $16(80)$ & $26(89.6)$ & $10(76.9)$ & 0.26 \\
\hline Beta-blockers, $n(\%)$ & - & $22(75.8)$ & $11(84.6)$ & 0.56 \\
\hline MRA, $n(\%)$ & - & $16(55.1)$ & $8(61.5)$ & 0.46 \\
\hline Loop diuretics, $n(\%)$ & - & $19(65.5)$ & $10(76.9)$ & 0.28 \\
\hline $\begin{array}{l}\text { Thiazide/thiazide-like } \\
\text { diuretics, } n(\%)\end{array}$ & $6(30)$ & $13(34.5)$ & $5(23.1)$ & 0.57 \\
\hline Statins (\%) & $14(70)$ & $20(68.9)$ & $10(76.9)$ & 0.86 \\
\hline \multicolumn{5}{|l|}{ Therapy for DM } \\
\hline Metformin & $19(95)$ & $29(100)$ & $13(100)$ & - \\
\hline DPPi-4 & $14(70)$ & $18(62.1)$ & $8(61.5)$ & 0.82 \\
\hline Sulfonylurea & $6(30)$ & $11(37.9)$ & $5(38.5)$ & \\
\hline
\end{tabular}

CHF: chronic heart failure; CAD: coronary artery disease; MI: myocardial infarction; CABG: coronary artery bypass grafting; PCI: percutaneous coronary intervention; SBP: systolic blood pressure; DBP: diastolic blood pressure; BMI: body mass index; GFR: glomerular filtration rate; ACEi: angiotensinconverting-enzyme inhibitors; ARA: angiotensin receptor antagonists; MRA: mineralocorticoid receptor antagonists; DPPi-4: dipeptidil peptidase-4 inhibitors.

and cardiac steatosis. Cardiac remodeling and impaired microvascular coronary perfusion contribute to the development of diastolic dysfunction $[3,4,11,12]$. High glucose level stimulates fibroblast proliferation and activates transcription and secretion of extracellular matrix proteins. Hyperglycemia also contributes to increased activity of local renin-angiotensinaldosterone system (RAAS) in the heart. This increased activity may promote profibrotic processes due to increase in the TGF$\beta$ expression and reactive oxygen species formation [13].
Most patients with T2DM and HF have comorbidities such as obesity, $\mathrm{AH}$, and accelerated atherosclerosis, which determining the complex clinical phenotype of $\mathrm{HF}$, mainly with LV EF $>40 \%$. The presented study included patients with T2DM, HF with midrange, and preserved LV EF, who have additional cardiometabolic risk factors for $\mathrm{HF}$ (increased $\mathrm{BMI}$ and $\mathrm{AH}$ ), and in more than half of the cases, the presence of IHD. It is well known that insulin resistance, metabolic syndrome, and T2DM are associated with an increase in 
TABLE 2: Echocardiographic parameters of patients, $\operatorname{Me}(25,75)$.

\begin{tabular}{|c|c|c|c|c|c|c|c|}
\hline \multirow[b]{2}{*}{ Parameters } & \multirow{2}{*}{$\begin{array}{c}\text { Group } 1 \text { without HF } \\
(n=20) \\
\operatorname{Me}[25 ; 75]\end{array}$} & \multirow{2}{*}{$\begin{array}{c}\text { Group 2 HFpEF } \\
(n=29) \\
\operatorname{Me}(25,75)\end{array}$} & \multirow{2}{*}{$\begin{array}{c}\text { Group } 3 \text { HFmrEF } \\
(n=13) \\
\operatorname{Me}(25,75)\end{array}$} & \multicolumn{4}{|c|}{$p$ value } \\
\hline & & & & (1 vs. 2 vs. 3 ) & (1 vs. 2 ) & (1 vs. 3 ) & (2 vs. 3 ) \\
\hline EF (Simpson), \% & $61(59,65)$ & $61(57,62)$ & $46(41,48)$ & $<0.001$ & 0.12 & $<0.001$ & $<0.001$ \\
\hline LA dimension, mm & $43(40,47)$ & $41(38,47)$ & $44(40,50)$ & 0.29 & 0.34 & 0.22 & 0.21 \\
\hline LA volume index, $\mathrm{ml} / \mathrm{m}^{2}$ & $33.5(30.0,38.7)$ & $42.0(37.1,46.4)$ & $48.3(38.0,59.0)$ & $<0.001$ & 0.003 & $<0.001$ & 0.23 \\
\hline E/e’avg & $7(8,9)$ & $11(9,13)$ & $11(9,14)$ & 0.006 & 0.009 & 0.005 & 0.4 \\
\hline PAP, mmHg & $26(19,30)$ & $24(18-29)$ & $26(20-31)$ & 0.57 & 0.37 & 0.61 & 0.18 \\
\hline LV dimension, $\mathrm{mm}$ & $49(46,53)$ & $47(45,51)$ & $50(46,52)$ & 0.4 & 0.49 & 0.43 & 0.13 \\
\hline $\mathrm{EDV}, \mathrm{ml}$ & $111(94.7,126.7)$ & $117(102,135)$ & $167(125,220)$ & 0.025 & 0.21 & 0.005 & 0.007 \\
\hline $\mathrm{LV}$ volume index, $\mathrm{ml} / \mathrm{m}^{2}$ & $50(45,63)$ & $59(49,68)$ & $66(59,74)$ & 0.08 & 0.13 & 0.03 & 0.037 \\
\hline $\mathrm{ESV}, \mathrm{ml}$ & $46(34,53)$ & $45(39,61)$ & $89(61,134)$ & 0.007 & 0.35 & 0.001 & 0.002 \\
\hline IVS, mm & $10(9,11)$ & $11(10,13)$ & $11.5(11,13)$ & 0.24 & 0.56 & 0.39 & 0.85 \\
\hline $\mathrm{PW}, \mathrm{mm}$ & $11(10,12)$ & $11(10,13)$ & $11(9,12)$ & 0.51 & 0.43 & 0.53 & 0.41 \\
\hline RWT & $0.44(0.41,0.46)$ & $0.53(0.49,0.57)$ & $0.52(0.5,0.55)$ & 0.009 & 0.007 & 0.01 & 0.77 \\
\hline LVMM, g & $197(177,244)$ & $252(207,335)$ & $271(228,401)$ & 0.01 & 0.07 & 0.034 & 0.28 \\
\hline LVM/BSA, g/m² & $105(91,119)$ & $118(94,142)$ & $155(97,196)$ & 0.023 & 0.27 & 0.02 & 0.17 \\
\hline LVM/height, g/m & $116(109,131)$ & $177(156.0,192.8)$ & $205.7(160.1,220.6)$ & 0.002 & 0.02 & 0.001 & 0.043 \\
\hline LVM/height, $\mathrm{g} / \mathrm{m}^{2.7}$ & $49(42,56)$ & $52.5(46.6,75.1)$ & $69.2(52.6,89.3)$ & 0.04 & 0.15 & 0.01 & 0.08 \\
\hline GLS avg, \% & $-19(-17,-20)$ & $-19(-17,-20)$ & $-16.5(-14,-19.0)$ & 0.016 & 0.91 & 0.041 & 0.041 \\
\hline
\end{tabular}

EF: ejection fraction; LV: left ventricle; ESV: end-systolic volume of the left ventricle; EDV: end-diastolic volume of the left ventricle; LVM: left ventricular myocardial mass; LA: left atrium; IVS: interventricular septum; PW: posterior wall of left ventricle; RWT: relative wall thickness of the left ventricle; BSA: body surface area.

myocardial mass and LV RWT [10] and predispose to the development of concentric LV hypertrophy. Therefore, we included patients with similar BMI and glycemic control. In our study, patients with T2DM and HFmrEF were characterized by increased values of EDV, indexed parameters of LVM, and higher values of Nt-proBNP compared with patients with HFpEF. This observation is consistent with the data of other authors, who conducted a comparative study of echocardiographic parameters in patients with HFpEF and HFmrEF [14]. Similar patterns of echocardiographic changes and NtproBNP concentrations were presented in other earlier studies; some authors consider HFmrEF as the initial stage of HFrEF [15]. An increase in LA size is an indicator of a longterm raising in LV filling pressure, and LA volume and function are predictors of an increase in brain natriuretic peptides in patients with HF $[14,16]$. We did not find any correlations between the parameters of diastolic dysfunction and the level of Nt-proBNP; however, a strong positive association with the marker of myocardial stress and the LA volume index confirms the association of the LA parameters with neurohormonal activation of the heart in T2DM patients with HFmrEF or HFpEF. Various aspects associated with NtproBNP from diagnostics to B-type natriuretic peptideguided therapy in different populations of patients with HF are actively studied [17-20].

Comparative studies of molecular biomarkers show that patients with HFmrEF have an intermediate biomarker profile associated with myocardial stress, inflammation, and fibrosis [21-23]. Despite the high incidence of T2DM in patients with HFpEF and HFmrEF in these studies, the data on the effect of glycemic status on the molecular biomarkers in these patients are lacking. In our study, we analyzed the wide range of biomarkers in patients with T2DM, multiple cardiometabolic risk factors, and stable HF with LV EF > 40\%.

sST2 is a member of the interleukin-1 receptor family and a marker of inflammation and myocardial stress. An increase in the concentrations of sST2 is observed in various cardiovascular diseases, visceral obesity, and T2DM [24]. The significantly lower variability for sST2 in comparison with NtproBNP makes it promising for assessing the prognosis of the survival of patients with HF. sST2 is considered as a new marker of cardiovascular events and adverse clinical outcomes, primarily associated with HF and IHD [19]. In our study, serum sST2 in the group with HFpEF did not differ from group with HFmrEF, which is consistent with Song et al. [25]. The lack of differences in serum sST2 concentrations between the control group and study groups can be explained by both the inclusion of patients with mild HF, receiving optimal drug therapy for $\mathrm{HF}$, and the probably low sensitivity of this marker in patients with visceral obesity and T2DM. Despite the prognostic value of sST2 in patients with HFmrEF, its predictive value in patients with concomitant T2DM is doubtful [22-25].

Galectin-3 is a mediator of cell adhesion, inflammation, and extracellular matrix formation both in the myocardium and in the vascular wall $[6,26]$. In the studied cohort of patients with T2DM, irrespective of the existence of HF, the increase of galectin-3 was revealed compared to the control group. This observation confirms the intensity of inflammation and fibrosis in patients with T2DM and additional multiple etiological factors of HF, such as AH and obesity, even at the preclinical stage of HF. According to our data, higher 


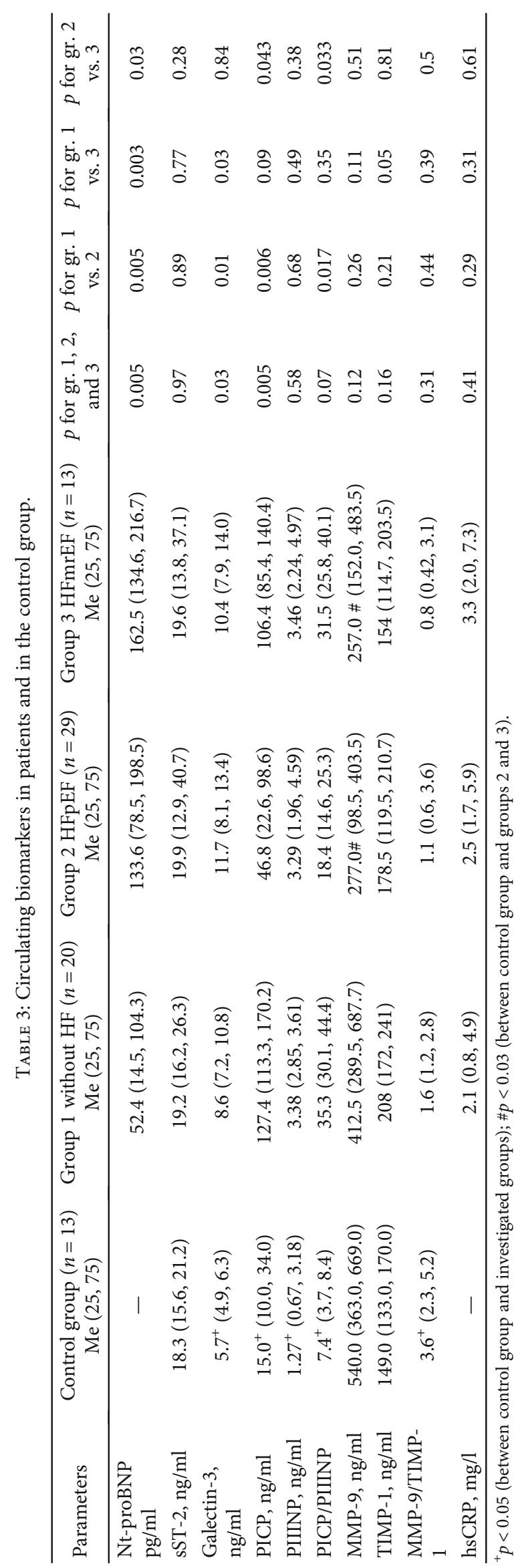


serum concentrations of this biomarker and a positive association with LVM in patients with HF confirm the role of galectin-3 in the pathogenesis of HF. However, we did not identify significant differences in galectin-3 concentrations between group with HFpEF and group with HFmrEF. Our data is consistent with another study of patients with HFmrEF and HFpEF; one-third of whom had T2DM and more severe HF [21]. An increase in serum galectin-3 is considered an adaptive response to inflammation and impaired glucose metabolism. However, the data on the predictive value of galectin- 3 in relation to other traditional biomarkers in T2DM patients with HF remain ambiguous [24, 27, 28].

According to histological studies, interstitial and perivascular fibrosis in T2DM is associated with an increase in type I collagen and type III collagen, regardless of coronary atherosclerosis and $\mathrm{AH}[29,30]$. In addition to reactive fibrosis, magnetic resonance imaging often visualizes areas of fibrosis due to asymptomatic coronary events in T2DM patients without MI [31]. These changes contribute to the further remodeling of the myocardium and the progression of HF. The predominant type of the collagen in the extracellular matrix determines by etiological factor of HF. In addition, quantitative and qualitative changes of fibrotic processes are dynamic, presenting different phenotypes of interstitial fibrosis over time, including chronic hyperglycemia [32]. An association between diabetes and an increase in the collagen composition were observed only in patients with HFrEF, according to results of endomyocardial biopsies in patients with T2DM complicated by HFpEF and HFrEF (LVEF < 45\%) in the absence of coronary atherosclerosis [29, 33]. Relevant circulating markers of fibrosis include PICP and PIIINP as biomarkers of types I and III collagen formation, respectively [34]. In a number of studies of patients with HF, endomyocardial biopsies have been shown a correlation between the serum PICP concentrations and the total collagen volume in patient with $\mathrm{AH}$, as well as between the PIIINP concentration and the volume of type III collagen in patients with more significant myocardial remodeling in IHD and dilated cardiomyopathy [35-38]. However, there are some controversial issues with these circulating biomarkers in patients with HF [34]. It should be taken into account that in vitro secretion of PICP and PIIINP by fibroblasts is influenced by level of glycemia, $\mathrm{AH}$, and obesity, which complicates their evaluation in patients with T2DM and HF [39-41]. There is a negative association of PIIINP with parameters of subclinical diastolic dysfunction in patients with T2DM in one study [42], while other authors showed a similar association in relation to PICP [43]. There are currently few studies of the described serum markers in HFmrEF, mainly in the meta-analysis of randomized clinical trials in patients with $\mathrm{LVEF}>40 \%$ [44]. In our study, we identified an increase in the concentrations of PICP and PIIINP in patients with T2DM, regardless of the presence of HF. The PICP levels and the PICIP/PIIINP ratio were significantly higher in the group with HFmrEF compared with the group with HFpEF. PICP/PIIINP ratio was shifted towards the marker of harder collagen in patients with HFmrEF compared to healthy individuals and patients with HFpEF. Furthermore, PIIINP concentration was associated with an increase in LV EDV in all patients with T2DM and HF. The obtained data confirm that a delicate balance between the synthesis and degradation of two types of collagen can determine the structural and functional changes in the myocardium in HF patients with impaired glycemic status. Similar patterns of changes in PICP and PIIINP were observed in the group without HF, which confirms the difficulties discussed above in interpreting the changes of circulating procollagen markers in patients with multiple cardiometabolic risk factors and other possible factors affecting the serum concentrations of these biomarkers. Taking into account the ambiguous data on the diagnostic significance of PICP and PIIINP depending on the etiology of HF, concomitant diseases affecting collagen metabolism, the obtained results of our research require further investigation [34].

Matrix metalloproteinases and their inhibitors play an important role in the exchange of the extracellular matrix, determining the collagen degradation processes. It has been shown that changes in the level of MMP-9, as well as TIMP-1, associated with nonspecific inflammation, and the balance between MMP-9 and TIMP-1 depend on the etiological cause and stage of the cardiovascular disease continuum $[26,34,45]$. Despite numerous evidences of an imbalance in the MMP-9 in T2DM, information about changes in the MMP-9 and TIMP-1 system is contradictory. As in the study by Lewandowski et al., we recorded a decrease in the serum concentration of MMP-9 in patients with T2DM compared with the control group, without reaching the threshold of significance [45]. Low levels of MMP-9 in the studied cohort of patients can be promoted by the drug effects of inhibitors of the renin-angiotensin-aldosterone system, statins, or antihyperglycemic therapy [46-48]. Interestingly, the level of MMP-9 decreased to the greater extent in patients with HF, although not significantly. At the same time, MMP9/TIMP-1 ratio significantly decreased in all groups of patients compared to the control group, especially in patients with more severe structural and functional heart remodeling. It can indicate the predominance of fibrotic processes in this group of patients. Negative association of TIMP-1 and LV EDV in patients with HF in our study confirms this assumption. The role of matrix metalloproteinases and their tissue inhibitors in the pathophysiology and prognosis in different HF phenotypes is not fully defined and continues to be studied. However, the MMP-9 expression is determined by the nature of the pathological process and the stage of HF [45].

\section{Conclusion}

There is a dynamic balance between the serum concentrations of biomarkers reflecting pro- and antifibrotic processes in patients with T2DM having additional risk factors of HF without and with clinical manifestations of HF in real clinical practice. The dynamics and direction of changes at various stages of the cardiovascular disease continuum in the serum fibrosis markers, MMP-9 and TIMP-1 systems, may reflect an increase in fibrotic and decrease in antifibrotic processes 
already at the preclinical stage of HF. At the same time, the changes found in the PICP and PIIINP system may indicate a shift in balance towards type I collagen synthesis in HFmrEF compared with HFpEF.

Further prospective studies on large samples using a multibiomarker panel, methods of multivariate statistics in patients with T2DM, and various HF phenotypes are needed.

\section{Research Limitations}

Our study has several limitations. A small sample size as well as the presence of possible confounding factors, including obesity phenotype and other factors not considered in the study, can influence the concentrations of the studied biomarkers of fibrosis. MMP-9 and TIMP-1 were measured in the serum, which can overestimate the concentrations of these biomarkers compared with the levels in plasma samples [43]. The protocol did not include patients with severe HF (III-IV functional class NYHA) and with HFrEF; however, it may be of scientific interest for a more complete understanding of the clinical phenotype of HFmrEF in patients with T2DM.

\section{Data Availability}

Research data can be available after contact by e-mail doctorlebedev11@gmail.com with corresponding author.

\section{Conflicts of Interest}

The authors declare that they have no conflicts of interest.

\section{Authors' Contributions}

We confirm that all the authors participated in the preparation of the manuscript.

\section{Acknowledgments}

This research was conducted with the support of the Russian Scientific Fund (grant number 17-7530052).

\section{References}

[1] F. Cosentino, P. J. Grant, V. Aboyans et al., "2019 ESC Guidelines on diabetes, pre-diabetes, and cardiovascular diseases developed in collaboration with the EASD," European Heart Journal, vol. 41, no. 2, pp. 255-323, 2020.

[2] I. Johansson, U. Dahlström, M. Edner, P. Näsman, L. Rydén, and A. Norhammar, "Type 2 diabetes and heart failure: characteristics and prognosis in preserved, mid-range and reduced ventricular function," Diabetes \& Vascular Disease Research, vol. 15, no. 6, pp. 494-503, 2018.

[3] T. Miki, S. Yuda, H. Kouzu, and T. Miura, "Diabetic cardiomyopathy: pathophysiology and clinical features," Heart Failure Reviews, vol. 18, no. 2, pp. 149-166, 2013.

[4] W. J. Paulus and C. Tschöpe, "A novel paradigm for heart failure with preserved ejection fraction: comorbidities drive myocardial dysfunction and remodeling through coronary microvascular endothelial inflammation," Journal of the American College of Cardiology, vol. 62, no. 4, pp. 263-271, 2013.
[5] F. T. Ageev and A. G. Ovchinnikov, "Heart failure with midrange ejection fraction: are there clinical reasons in introduction of this new group as a distinct entity?," Kardiologiia, vol. 58, no. 12S, pp. 4-10, 2018.

[6] M. Sarhene, Y. Wang, J. Wei et al., "Biomarkers in heart failure: the past, current and future," Heart Failure Reviews, vol. 24, no. 6, pp. 867-903, 2019.

[7] P. Ponikowski, A. A. Voors, S. D. Anker et al., "2016 ESC Guidelines for the diagnosis and treatment of acute and chronic heart failure: the task force for the diagnosis and treatment of acute and chronic heart failure of the European Society of Cardiology (ESC). Developed with the special contribution of the Heart Failure Association (HFA) of the ESC," European Heart Journal, vol. 37, no. 27, pp. 2129-2200, 2016.

[8] B. Williams, G. Mancia, W. Spiering et al., "2018 ESC/ESH Guidelines for the management of arterial hypertension," European Heart Journal, vol. 39, no. 33, pp. 3021-3104, 2018.

[9] M. Singh, A. Sethi, A. K. Mishra, N. K. Subrayappa, D. D. Stapleton, and P. A. Pellikka, "Echocardiographic imaging challenges in obesity: guideline recommendations and limitations of adjusting to body size," Journal of the American Heart Association, vol. 9, no. 2, p. e014609, 2020.

[10] T. H. Marwick, T. C. Gillebert, G. Aurigemma et al., "Recommendations on the use of echocardiography in adult hypertension: a report from the European Association of Cardiovascular Imaging (EACVI) and the American Society of Echocardiography (ASE)," Journal of the American Society of Echocardiography, vol. 28, no. 7, pp. 727-754, 2015.

[11] H. C. Kenny and E. D. Abel, "Heart failure in type 2 diabetes mellitus," Circulation Research, vol. 124, no. 1, pp. 121-141, 2019.

[12] J. M. McGavock, I. Lingvay, I. Zib et al., "Cardiac steatosis in diabetes mellitus: a $1 \mathrm{H}$-magnetic resonance spectroscopy study," Circulation, vol. 116, no. 10, pp. 1170-1175, 2007.

[13] G. Jia, M. A. Hill, and J. R. Sowers, "Diabetic Cardiomyopathy: An Update of Mechanisms Contributing to This Clinical Entity," Circulation Research, vol. 122, no. 4, pp. 624-638, 2018.

[14] L. al Saikhan, A. D. Hughes, W. S. Chung, M. Alsharqi, and P. Nihoyannopoulos, "Left atrial function in heart failure with mid-range ejection fraction differs from that of heart failure with preserved ejection fraction: a 2D speckle-tracking echocardiographic study," European Heart Journal Cardiovascular Imaging, vol. 20, no. 3, pp. 279-290, 2019.

[15] C. S. P. Lam and S. D. Solomon, "The middle child in heart failure: heart failure with mid-range ejection fraction (4050\%)," European Journal of Heart Failure Supplements, vol. 16, no. 10, pp. 1049-1055, 2014.

[16] M. Baba, K. Yoshida, and M. Ieda, "Clinical applications of natriuretic peptides in heart failure and atrial fibrillation," International Journal of Molecular Sciences, vol. 20, no. 11, p. 2824, 2019.

[17] G. Gallo, F. Bianchi, M. Cotugno, M. Volpe, and S. Rubattu, "Natriuretic peptides, cognitive impairment and dementia: an intriguing pathogenic link with implications in hypertension," Journal of Clinical Medicine, vol. 9, no. 7, p. 2265, 2020.

[18] D. Hakuno, T. Fukae, M. Takahashi et al., "Combinations of cardiac and non-cardiac predictors for prognoses in patients with acute heart failure," European Heart Journal - Quality of Care and Clinical Outcomes, vol. qcz059, pp. 1-14, 2019.

[19] K. Ejiri, T. Miyoshi, H. Kihara et al., "Effect of luseogliflozin on heart failure with preserved ejection fraction in patients with 
diabetes mellitus," Journal of the American Heart Association, vol. 9, no. 16, article e015103, 2020.

[20] M. Pufulete, R. Maishman, L. Dabner et al., "B-type natriuretic peptide-guided therapy for heart failure (HF): a systematic review and meta-analysis of individual participant data (IPD) and aggregate data," Systematic Reviews, vol. 7, no. 1, p. 112, 2018.

[21] P. Moliner, J. Lupón, J. Barallat et al., "Bio-profiling and bioprognostication of chronic heart failure with mid-range ejection fraction," International Journal of Cardiology, vol. 257, pp. 188-192, 2018.

[22] J. Tromp, M. A. F. Khan, R. J. Mentz et al., "Biomarker profiles of acute heart failure patients with a mid-range ejection fraction," JACC: Heart Failure, vol. 5, no. 7, pp. 507-517, 2017.

[23] O. Chioncel, M. Lainscak, P. M. Seferovic et al., "Epidemiology and one-year outcomes in patients with chronic heart failure and preserved, mid-range and reduced ejection fraction: an analysis of the ESC Heart Failure Long-Term Registry," European Journal of Heart Failure Supplements, vol. 19, no. 12, pp. 1574-1585, 2017.

[24] A. E. Berezin, "Prognostication of clinical outcomes in diabetes mellitus: emerging role of cardiac biomarkers," Diabetes and Metabolic Syndrome: Clinical Research and Reviews, vol. 13, no. 2, pp. 995-1003, 2019.

[25] Y. Song, F. Li, Y. Xu et al., "Prognostic value of sST2 in patients with heart failure with reduced, mid-range and preserved ejection fraction," International Journal of Cardiology, vol. 304, pp. 95-100, 2020.

[26] N. Ibrahim and J. Januzzi, "Established and emerging roles of biomarkers in heart failure," Circulation Research, vol. 123, no. 5, pp. 614-629, 2018.

[27] R. A. de Boer, D. J. van Veldhuisen, R. T. Gansevoort et al., "The fibrosis marker galectin-3 and outcome in the general population," Journal of Internal Medicine, vol. 272, no. 1, pp. 55-64, 2012.

[28] N. Alonso, J. Lupón, J. Barallat et al., "Impact of diabetes on the predictive value of heart failure biomarkers," Cardiovascular Diabetology, vol. 15, no. 1, p. 151, 2016.

[29] I. Russo and N. G. Frangogiannis, "Diabetes-associated cardiac fibrosis: cellular effectors, molecular mechanisms and therapeutic opportunities," Journal of Molecular and Cellular Cardiology, vol. 90, pp. 84-93, 2016.

[30] T. J. Regan, M. M. Lyons, S. S. Ahmed et al., "Evidence for cardiomyopathy in familial diabetes mellitus," The Journal of Clinical Investigation, vol. 60, no. 4, pp. 885-899, 1977.

[31] R. Y. Kwong, H. Sattar, H. Wu et al., "Incidence and prognostic implication of unrecognized myocardial scar characterized by cardiac magnetic resonance in diabetic patients without clinical evidence of myocardial infarction," Circulation, vol. 118, no. 10, pp. 1011-1020, 2008.

[32] A. González, E. B. Schelbert, J. Díez, and J. Butler, "Myocardial interstitial fibrosis in heart failure: biological and translational perspectives," Journal of the American College of Cardiology, vol. 71, no. 15, pp. 1696-1706, 2018.

[33] L. van Heerebeek, N. Hamdani, M. L. Handoko et al., "Diastolic stiffness of the failing diabetic heart: importance of fibrosis, advanced glycation end products, and myocyte resting tension," Circulation, vol. 117, no. 1, pp. 43-51, 2008.

[34] B. López, A. González, S. Ravassa et al., "Circulating biomarkers of myocardial fibrosis," Journal of the American College of Cardiology, vol. 65, no. 22, pp. 2449-2456, 2015.
[35] B. López, R. Querejeta, A. González, M. Larman, and J. Díez, "Collagen cross-linking but not collagen amount associates with elevated filling pressures in hypertensive patients with stage C heart failure: potential role of lysyl oxidase," Hypertension, vol. 60, no. 3, pp. 677-683, 2012.

[36] R. Querejeta, B. López, A. González et al., "Increased collagen type I synthesis in patients with heart failure of hypertensive origin: relation to myocardial fibrosis," Circulation, vol. 110, no. 10, pp. 1263-1268, 2004.

[37] B. López, R. Querejeta, A. González, E. Sánchez, M. Larman, and J. Díez, "Effects of loop diuretics on myocardial fibrosis and collagen type I turnover in chronic heart failure," Journal of the American College of Cardiology, vol. 43, no. 11, pp. 20282035, 2004.

[38] G. Klappacher, P. Franzen, D. Haab et al., "Measuring extracellular matrix turnover in the serum of patients with idiopathic or ischemic dilated cardiomyopathy and impact on diagnosis and prognosis," The American Journal of Cardiology, vol. 75, no. 14, pp. 913-918, 1995.

[39] S. P. Levick and A. Widiapradja, "The diabetic cardiac fibroblast: mechanisms underlying phenotype and function," International Journal of Molecular Sciences, vol. 21, no. 3, p. 970, 2020.

[40] Y. Benazzoug, C. Borchiellini, J. Labat-Robert, L. Robert, and P. Kern, "Effect of high-glucose concentrations on the expression of collagens and fibronectin by fibroblasts in culture," Experimental Gerontology, vol. 33, no. 5, pp. 445-455, 1998.

[41] W. Kosmala, M. Przewlocka-Kosmala, H. Szczepanik-Osadnik, A. Mysiak, and T. H. Marwick, "Fibrosis and cardiac function in obesity: a randomised controlled trial of aldosterone blockade," Heart, vol. 99, no. 5, pp. 320-326, 2013.

[42] C. Jellis, J. Wright, D. Kennedy et al., “Association of imaging markers of myocardial fibrosis with metabolic and functional disturbances in early diabetic cardiomyopathy," Circulation. Cardiovascular Imaging, vol. 4, no. 6, pp. 693-702, 2011.

[43] S. H. Ihm, H. J. Youn, D. I. Shin et al., "Serum carboxyterminal propeptide of type I procollagen (PIP) is a marker of diastolic dysfunction in patients with early type 2 diabetes mellitus," International Journal of Cardiology, vol. 122, no. 3, pp. e36-e38, 2007.

[44] Y. Xiang, W. Shi, Z. Li et al., "Efficacy and safety of spironolactone in the heart failure with mid-range ejection fraction and heart failure with preserved ejection fraction: a meta-analysis of randomized clinical trials," Medicine (Baltimore), vol. 98, no. 13, article e14967, 2019.

[45] T. Morishita, H. Uzui, Y. Mitsuke et al., "Association between matrix metalloproteinase- 9 and worsening heart failure events in patients with chronic heart failure," ESC Heart Failure, vol. 4, no. 3, pp. 321-330, 2017.

[46] K. C. Lewandowski, E. Banach, M. Bieńkiewicz, and A. Lewiński, "Matrix metalloproteinases in type 2 diabetes and non-diabetic controls: effects of short-term and chronic hyperglycaemia," Archives of Medical Science, vol. 2, no. 2, pp. 294-303, 2011.

[47] W. D. Li, N. P. Li, D. D. Song, J. J. Rong, A. M. Qian, and X. Q. $\mathrm{Li}$, "Metformin inhibits endothelial progenitor cell migration by decreasing matrix metalloproteinases, MMP-2 and MMP-9, via the AMPK/mTOR/autophagy pathway," International Journal of Molecular Medicine, vol. 39, no. 5, pp. 1262-1268, 2017.

[48] C. S. Ceron and M. R. Luizon, "Plasma matrix metalloproteinases in coronary artery disease patients," European Journal of Clinical Investigation, vol. 46, no. 1, pp. 104-105, 2016. 